\title{
Communication
}

\section{Modification of monocyte count and subsets during human cytomegalovirus replication in vivo and glucocorticoid therapy}

\author{
Przemyslaw Zdziarski ${ }^{1,2,3}$, Andrzej Gamian ${ }^{3}$ \\ 1 Department of Clinical Immunology and Hospital Infection Control Team, Lower Silesian Center, \\ Wroclaw \\ 2 Military Institute WITI Wroclaw; 136 Obornicka Str., Wroclaw, Poland \\ 3 Hirszfeld Institute of Immunology and Experimental Therapy, Polish Academy of Sciences \\ * For correspondence P.O.Box 1818 50-385 Wrocław-46, Poland \\ Przemyslaw Zdziarski (ORCID 0000-0002-5692-4734) zdziarski@oil.org.pl
}

\section{Abstract}

It has recently been discovered that mere cell contact by human cytomegalovirus (CMV) particles leads to profound modulation of cellular gene expression. Reduced monocyte human leukocyte antigen (HLA-DR) expression is a novel biomarker of severity and outcome in many diseases. Modulation of CD14 protein by CMV was shown in vitro, but little is known about the phenomenon in vivo (during active cytomegalovirus disease).

Therefore, we investigated monocyte CD14 and HLA-DR expression in CMV infected patients in relation to logarithmic phase of infectious process. Samples from patients with active CMV replication (exponential growth of CMV viremia) were tested. After CD45/SSC gating monocyte CD14 and HLA-DR expression were determined by double-color flowcytometry.

Significant monocytosis and poor correlation between CMV replication and CD14+HLADR(-) cells prompted CD14 investigation. During logarithmic phase of CMV infection increased count and percentage of CD14low monocytes were observed which correlated with viral replication in several clinical situations except when there was a rapid recovery without relapse. Furthermore, most of CD14low monocytes are HLA-DR+. The increase of CD14low monocytes is also observed under the influence of high dose of glucocorticoids (20 mg of dexamethasone).

The reduction in CD14 induced by CMV and dexamethasone indicates that the monocyte balance is disturbed between the classical and non-classical phenotype. A high percentage of CD14lowHLA-DR+ probably gives rise to adaptive and a decrease of innate immune response. In light of the logarithmic increase of viral load (with exponent between 3,23 and $5,77)$, high monocytosis above $1200 / \mu 1$ is a hallmark of CMV replication. 
Keywords: cytomegalovirus (CMV), definitions of cytomegalovirus disease, logarithmic phase infection; innate immune response, monocytes, monocytosis, CD14, non-classical monocytes, HLA-DR, adaptive immune response, interferon

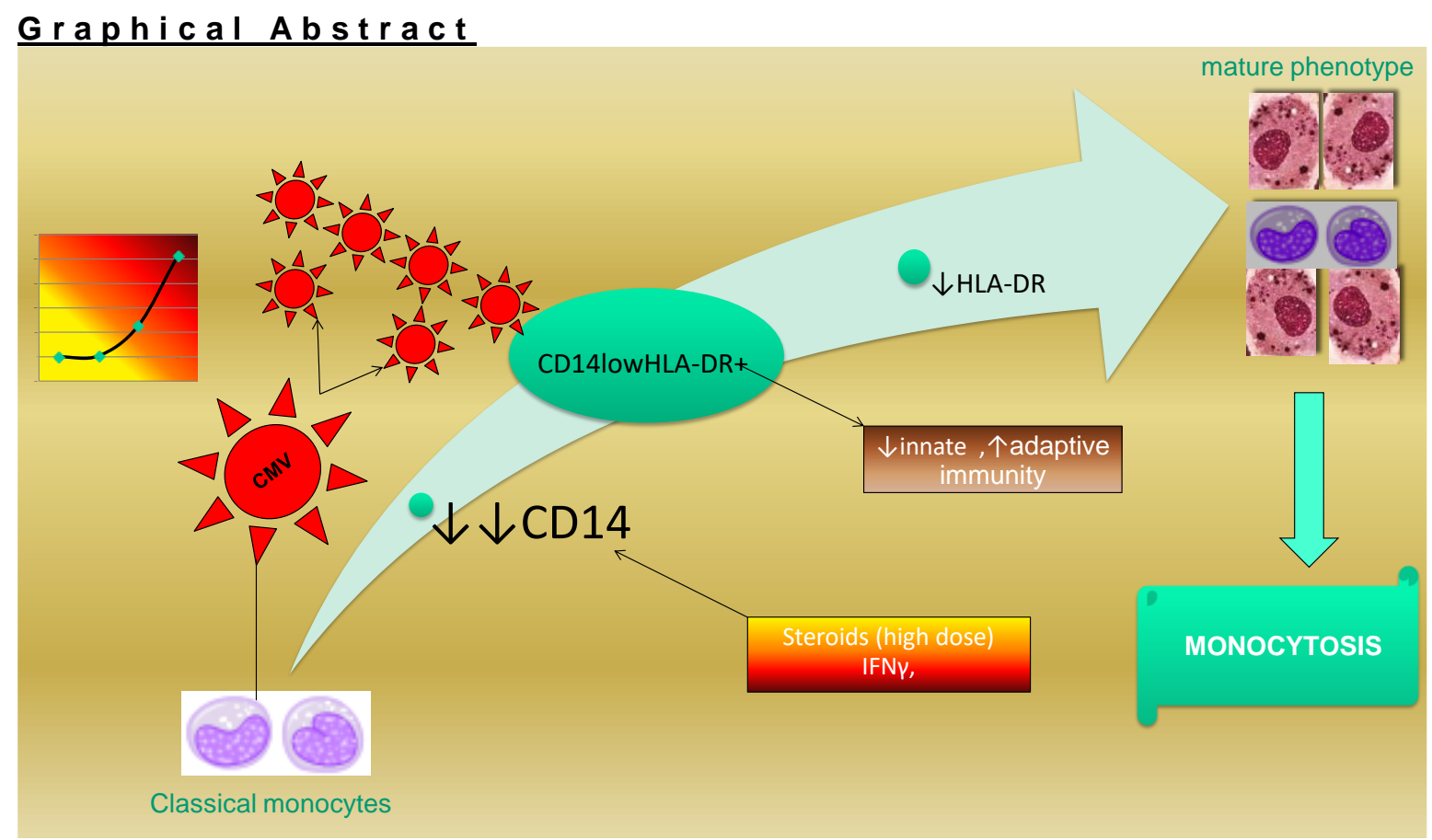

\section{Introduction}

Research on innate immune signaling and regulation has recently focused on pathogen recognition receptors (PRRs) and their signaling pathways. Although the adaptive immune response to $\mathrm{CMV}$ is a well described phenomenon, in clinical situation innate immune response precedes antigen presentation, lymphocyte recruitment and effector immune response. CD14 is a crucial co-receptor for Toll-like receptors (TLR 1,2,4,6) [1]. Specific large-scale and time-consuming experimental studies for appropriate explanation of CMV infectious process were conducted, but the clinical situation is more complicated and difficult in the interpretation [2,3]. Firstly, it is impossible to exclude all other causes of the clinical symptomatology described as CMV syndrome, patients receive several immunomodulatory drugs (e.g. steroids) and intra-host CMV diversity may be the source of atypical clinical manifestation [4]. Secondly, in vitro studies use known count of virions and specific CMV strains that are propagated on granulocyte-macrophage progenitor cells. In addition, reactivation of the virus is induced in experimentally infected granulocyte-macrophage progenitors by cocultivation with permissive cells or by treatment with proinflammatory cytokines [5]. Thirdly, the single positive result of polymerase chain reaction (PCR) may be difficult to interpret with latent viruses such as CMV. All patients previously infected will have a virus present in blood or tissue, irrespective of whether they have a disease or not. It is 
generally found that patients with active CMV have a much higher viral load, but appropriate cut-off level has not been universally described [6]. It is crucial that the detection of virus, antigen, or DNA with very sensitive methods in blood does not mean that CMV is currently in replicating phase [5]. Therefore, there is a significant gap of knowledge. Contrary to rapid development of molecular background, basic understanding of physiology, symptomatology and differential diagnosis of CMV disease is still an open question [3,7,8]. Unfortunately, CMV reactivation is arbitrarily defined as CMV viremia $>1000$ copies $/ \mathrm{mL}$ in spite of the fact that viral particles may be in free form or within leukocytes and deep leukopenia may affect the absolute result [9]. Such studies show ambiguous results with nearly the same prevalence of CMV reactivation and no CMV infection in recipient positive donor-negative constellation (R+/D-) i.e. $4.3 \%$ and $4.2 \%$, respectively. Furthermore, CMV disease defined in this way (CMV copies> 1000 and pneumonitis or gastrointestinal disease) was observed only in the case of the $\mathrm{D}+\mathrm{R}+$ constellation. No cytomegalovirus disease was observed in $\mathrm{D}-\mathrm{R}-$ or $\mathrm{D}-\mathrm{R}+$ pairs, contrary to the accepted rule, that patients undergoing allo-HSCT, the use of a CMV (-) donor to a CMV (+) patient (R+/D-) have been associated with an increased risk for nonrelapse mortality and decreased survival [10]. Other infectious causes of pneumonitis or colitis in such immunodeficient patients were not analyzed and no patients with CMV disease developed relapse [9]. For the development of CMV disease not only the size of the viral load is crucial, but also the parameters of the host, such as $\operatorname{IgG}$, leukocyte level, especially lymphopenia [11]. It prompted the use with $\geq 100 \mathrm{CMV}$ copy numbers $/ 10^{5}$ per nucleated blood cells, when the number of CMV genomic copies was calculated and related to the $\beta$ actin copies proportionally referring to $10^{5}$ white blood cells (WBCs) with calibration to a standard WHO calibrator [12]. Furthermore, although CMV mononucleosis diagnosed by reactive lymphocytes under the influence of antigen presenting cells, little attention has been paid to the function of monocytes. Relative monocytosis is usually observed in a chronic disease such as hematologic malignancy, protozoan infection, tuberculosis, sarcoidosis. Contrary to relative, the absolute monocytosis is therefore a rarely observed phenomenon in the human clinic [13]. The absolute number of monocytes and changes in several fractions are a derivative of their marrow production and fast (within a few days) migration into tissues. Laboratory norms for monocytosis are evolving with a significant spread between values above 500 or 1200/ $\mu 1$ (Wallach's Interpretation of Diagnostic Tests Ninth and Tenth editions, respectively) [13]. Contrary to lymphocyte subset, the sequential analysis of monocyte counts and subsets is not analyzed in CMV disease. Furthermore, because of the use of different antibodies for monocyte identification, the nomenclature of monocytes in human blood has 
become quite confusing due to the existence of several subpopulations and CD14 use as an exclusive monocyte indicator [14]. Although it is the most stable marker of monocyte lineage, it can be down-regulated completely by interleukin-4 [15]. It prompts further use of both: morphological and cytometric techniques of monocyte counting. In the present study, monocytes were monitored in patients who developed CMV replication by sequential counting, testing of the surface CD14 and HLA-DR expression. Due to the nomenclature and divergent conclusions of previous publications, an attempt was made to standardize the examined patients. Active phase virus replication was demonstrated by exponential growth of blood viremia.

\section{2. $\underline{\text { Results }}$}

In preliminary study the sequential analysis of CMV-viral load showed poor or low correlation with clinical manifestation, total monocyte count and subsets. The correlation coefficients between viremia and 1) monocytosis, 2) CD14+, 3) CD14low, 4) CD14lowHLA-DR+, 5) CD14lowHLA-DR(-) cells were $\mathrm{r}^{2}=0.3233 ; 0.2140 ; 0.3501$ 0.0372 and -0.1468 , respectively. Interestingly, under the influence of viremia growth the increase was observed for total monocytosis and CD14low cells, while regression lines for the remaining subtypes were horizontal or with slight decrease (Figure 1).. 


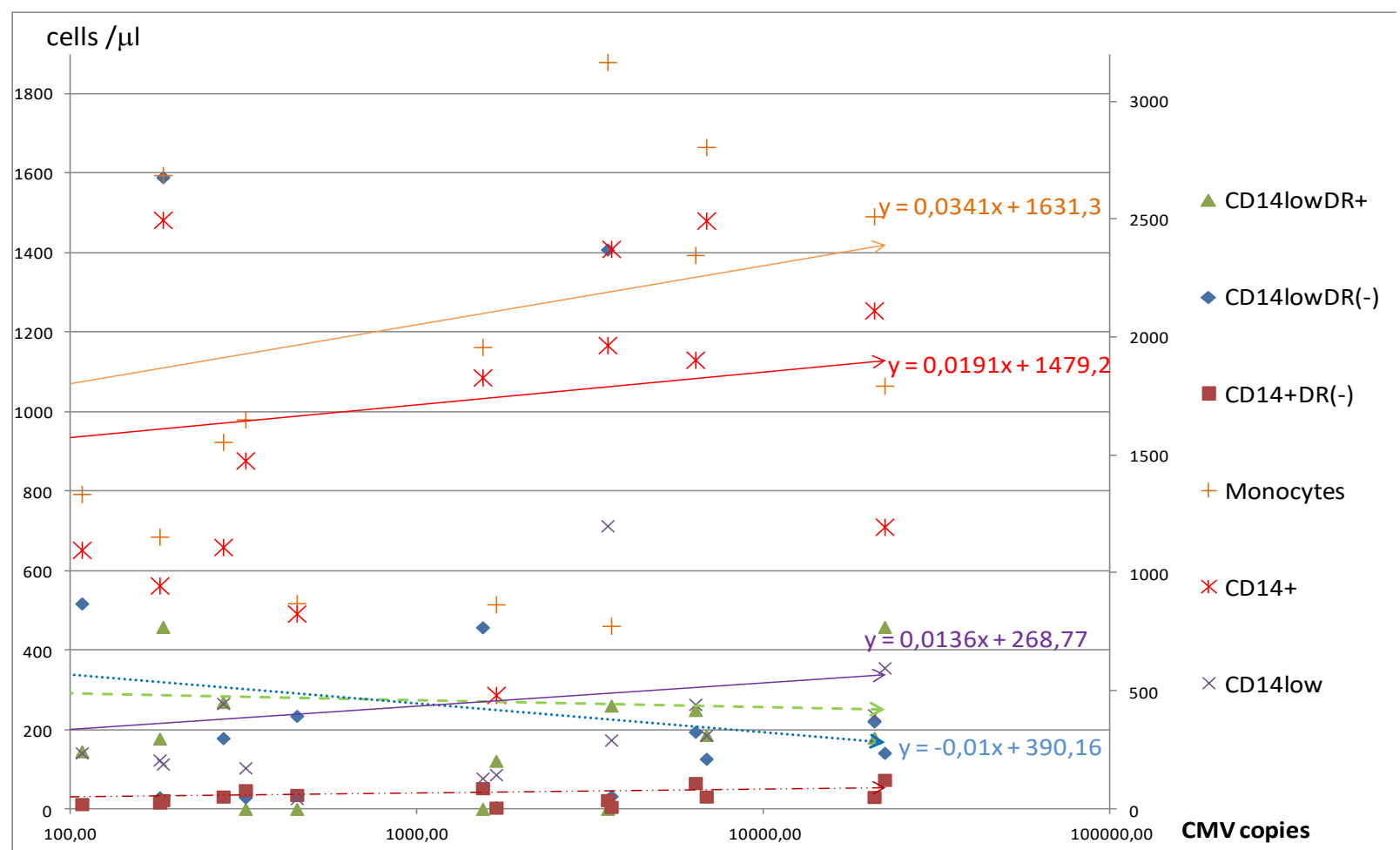

Figure 1. Regression and relationship between monocyte level and CMV viremia - presented on vertical axis in logarithmic scale. Monocyte, CD14+ and CD14low counts are expressed on right y axis.

Only the regression line of absolute monocyte, CD14+ classical and CD14low non-classical levels showed increasing tendency with nearly the same gradient, whereas correlations with viremia were poor. The dispersion of the results is significant: with low viral load $(<1000)$ when high monocytosis was sometimes observed (in the range of 900-1500).

Noteworthy, despite careful observation of patients, results about 1000 copies of the virus were observed intermittently. Contrary to total monocyte and subtype count the viremia level did not form continuous data. In the range of 500-1500 CMV copies a clear gap was observed. It indicates that, in contrast to the absolute value of CMV copy number, the exponential increase of viral load in timeline is a good measure of replication.

Furthermore, many patients showed stable CMV-viremia $\geq 100 \mathrm{CMV}$ copies $/ 10^{5}$ cells and they were subsequently treated with preemptive regimen of ganciclovir or valganciclovir, because they were designated as having clinically significant CMV risk as described in the analysis from our center [12]. Ganciclovir therapy with myelotoxic effect causes modification of marrow precursors and monocytosis. Therefore the patients were disqualified. Minority of patients $(16 / 160)$ had low viral load values that did not require treatment, followed by exponential increases of viremia in the subsequent analysis (see Materials and methods). When in such patients the exponential function of viremia was analyzed with base $=10$ (common logarithm) the exponent $\mathrm{x}$ was between 3.23 and 5.77. The highest exponent value corresponded with situation when CMV copy number was higher than WBC count. 


\subsection{Monocyte analysis by various techniques during exponential CMV replication. Significant monocvtosis as a hallmark}

In preliminary research simple gating step for forward and side scatter (FSC/SSC) for monocyte analysis was not useful in our patients (data not shown). Therefore, cytometric method based on CD45/SSC was used. It showed a very high correlation with the results obtained with the hematology analyzer. Both techniques were slightly less correlated with manual counting. The correlation coefficient between the two cytometry-based techniques was $r^{2}=0,97$, but interestingly the highest correlation was observed between hematology analyzer and CD45/SSC gating (Table 1).

\begin{tabular}{l|c|c|c}
\hline & Analyzer & CD45/SSC & CD14 \\
\hline Manual & 0,895264 & 0,932088 & 0,92599 \\
\hline Analyzer & & 0,986452 & 0,953596 \\
\hline CD45/SSC & & & 0,971847 \\
\hline
\end{tabular}

Table 1. Relationship between four most popular methods of monocyte analysis and counting. The Pearson $\mathbf{r}^{2}$ coefficients are presented.

Two techniques are based on morphology of monocytes

a) manual microscopic analysis of slides from May-Grünwald-Giemsa stain (MGG smears)

b) hematology analyzer contrary to two cytometric methods (CD14-based or CD45/SSC)

Our analysis also showed good correlation and concordance between cell count based on WBC enumeration and two cytometric approaches to monocyte definition (1) CD45vs.SSc gating (2) CD14++ mononuclear cells. Manual counting deviated from the other techniques (Table 1). No significant difference was observed between manual, hematology analyzer and CD45/SSC-based method, but unfortunately CD14-based flow cytometric method underestimated the monocyte level (Figure 2). 


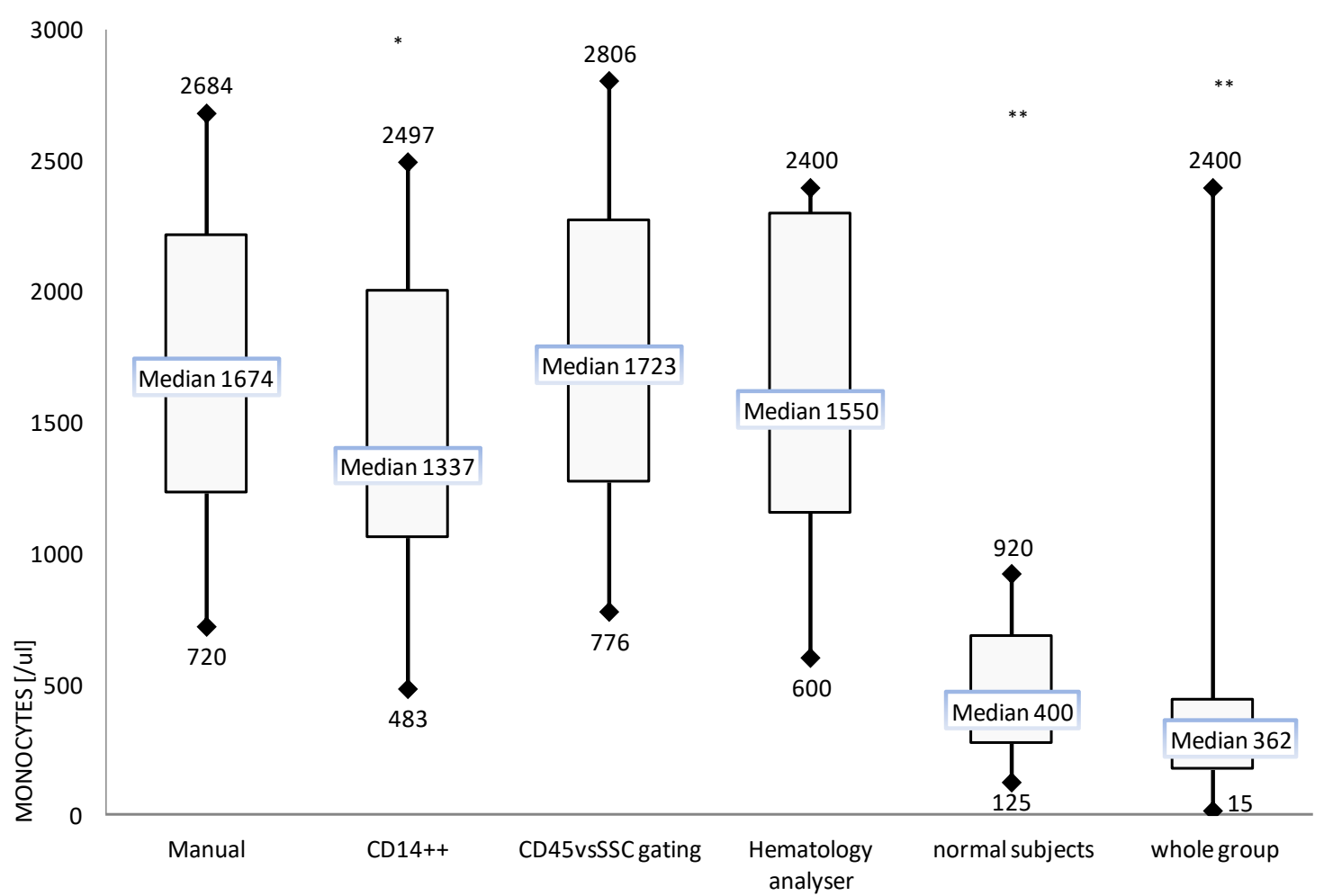

Figure 2. Box and whisker plot for the monocyte level in patients with CMV infection in exponential phase of infectious process.

Although significant monocytosis was observed, median value of CD14+ monocytes was significantly lower than monocyte level assessed by other techniques. For comparison there was presented the monocyte level in CMVsero-positive normal subjects without active replication (latent infection). Noteworthy, when we look at monocyte level in total group of 160 patients with viremia (in different phase of CMV disease) the median monocyte level was comparable with normal CMV-positive subjects without CMV disease. The data of normal subjects and of the whole group were obtained with hematology analyzer.

The correlation between the four methods of monocyte counting and viremia level was strong ( $\mathrm{r} 2=0.61214957$, $0.572744268,0.680241725$ and 0.662373669 for manual, CD14 or CD45-based and hematology analyser, respectively).

Contrary to simple correlation presented in Table 1, when we look at the relationship between monocyte count and linear regression, significantly lower values were observed when monocytes were defined as CD14 ++ mononuclear cells. The scatterplot of the changes in monocyte count is presented in Figure 3. 


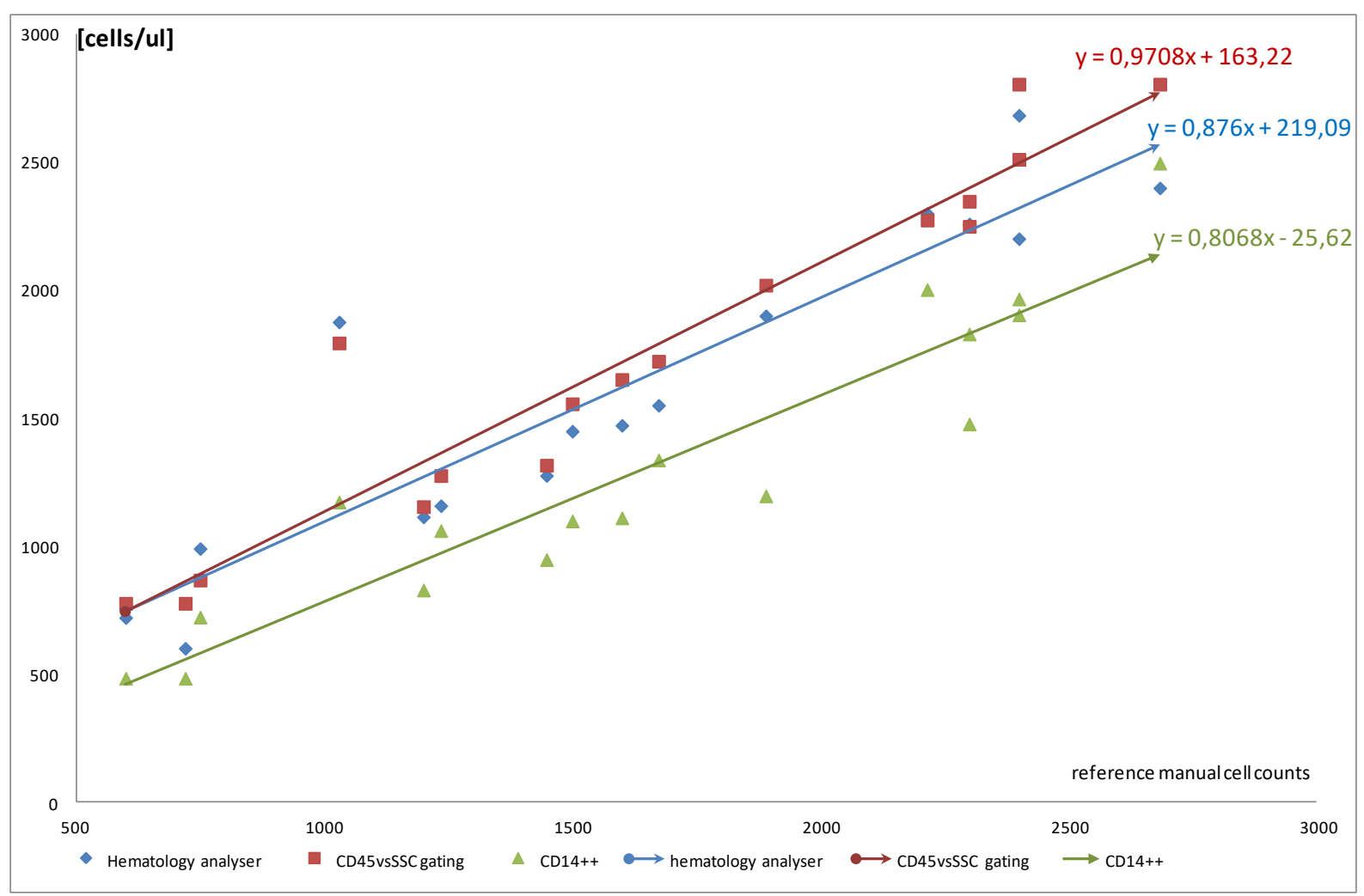

Figure 3 Regression and relationship between monocyte count obtained in manual reference method ( $x$ axis) and two flow methods 1) hematology analyzers 2) cytometry with CD45xSSC gating or 3) CD14++ cells presented on y axis.

High accuracy was observed, but Pearson coefficient was higher between two flow methods than between manual and autoanalyzer or cytometry methods (Table 1). The discrepancy was observed especially in patients with high monocytosis. However, CD14+ mononuclear cells showed good correlation with manual counting but absolute values of CD14 + monocytes were approximately 250 cells / $\mu$ l lower than in the hematology determinations (the trend lines were nearly parallel). Even greater difference was observed between CD14+monocytes and the gated CD45 + / SSC or by manual method (Figure2).

Both morphological and cytometric techniques of monocyte counting showed significant monocytosis during active CMV infection with exponential growth of CMV-viremia. To compare, the median monocyte level of sero-positive patients without active CMV replication was about 1000/ $\mu$ l lower (Figure 2). Non-selected whole group of 160 patients with positive CMV results showed high dispersion of monocyte level and comparable with healthy subjects median value.

\subsection{Blood monocvtes phenotype during exponential growth of CMV blood viremia.}

Typical monocytes CD14+HLA-DR+ were the majority of cells, however, CD14low involvement was noticeable and most of them expressed human leukocyte antigen (HLADR). HLA-DR negative cells were a marginal pool, although most of them were CD14low 
cells (Figure 4A). Absolute peripheral blood monocytosis (Figure 1-3) during CMV replication was also observed in the percentages (Figure 4B). 


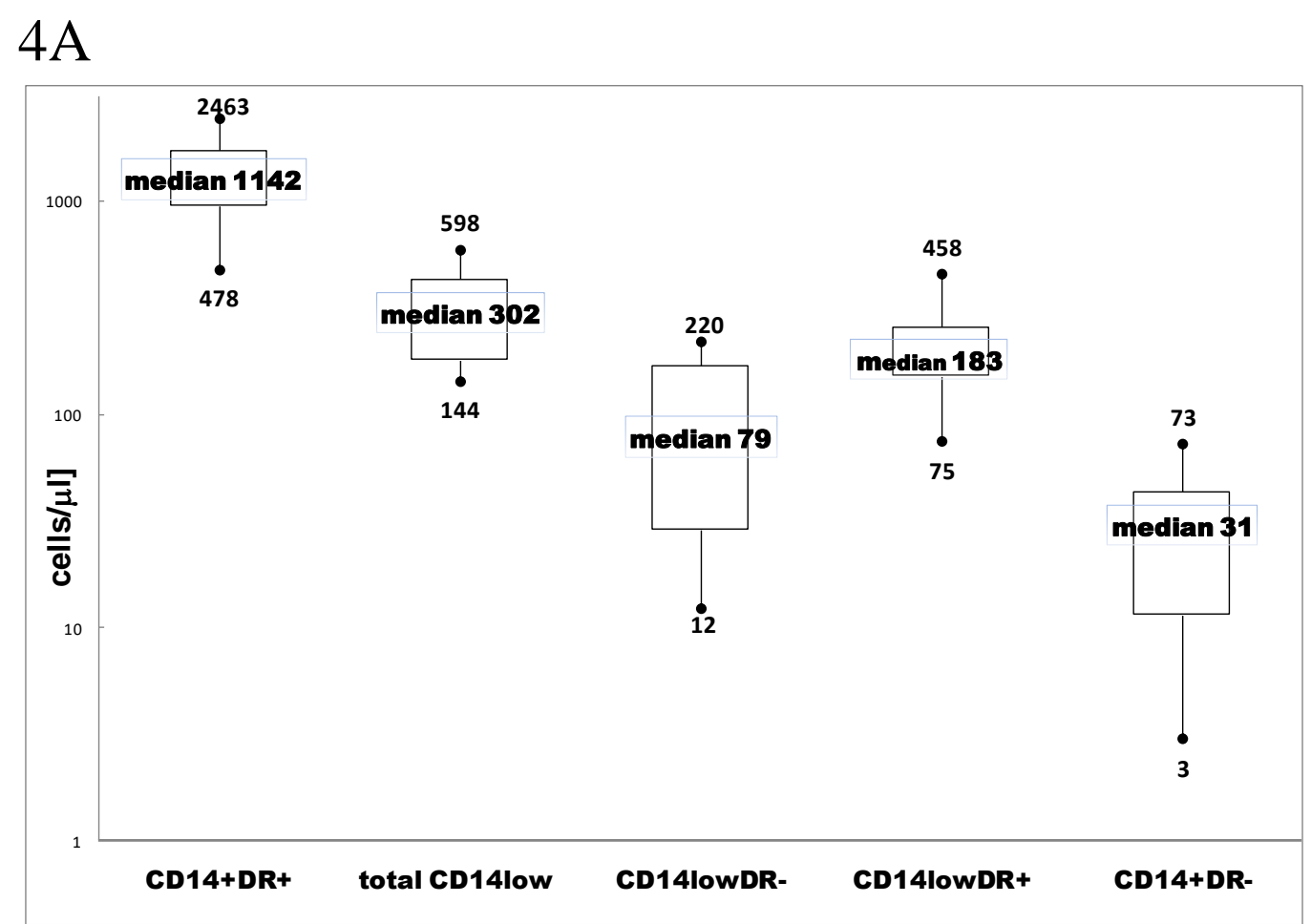

\section{B}

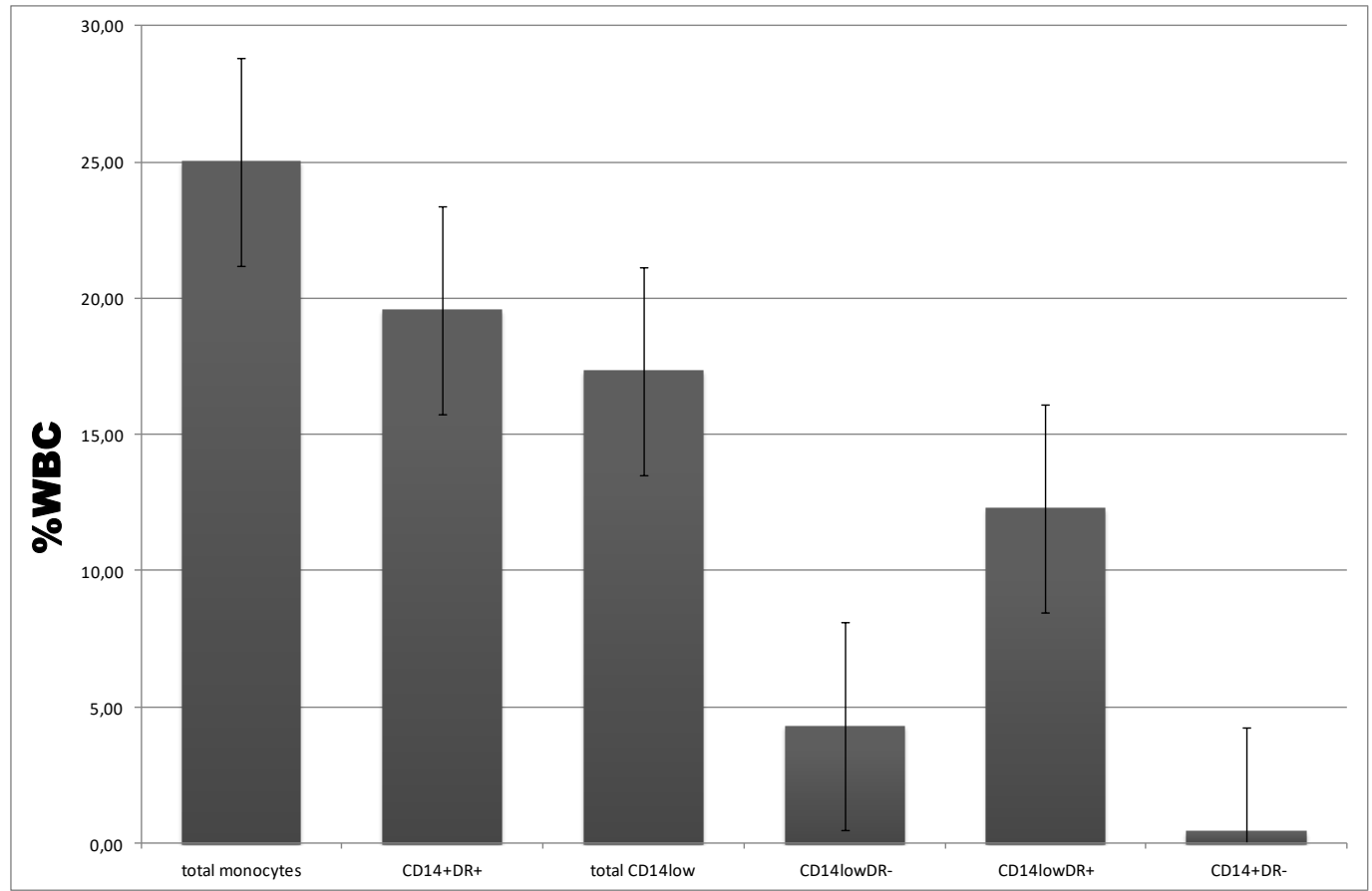

Figure 4 Blood monocytes subsets diversity during exponential growth of CMV blood viremia.

4A Box and whisker plot for the data from patients with active replication when exponential increase of CMV copies/ $\mu \mathrm{l}$ was observed. Values (logarithmic scale) represent the numbers of monocytes per $1 \mu 1$ measured at the same time. CD14low monocytes represent the population of monocytes with usually retained HLA-DR expression (median 183/ $\mu \mathrm{l}$ ). Data are presented as absolute numbers of monocyte subsets on a logarithmic scale 4B Median values of percentage of peripheral blood leukocytes (\%WBC) were presented. Significant relative monocytosis was observed in up to $25 \%$ of white blood cells. High percentage of monocytes in white blood cells was caused by an increase in the absolute number (Figure 1 and $3 \mathrm{~A}$ ) as well as significant lymphopenia in patients with immunodeficiency. 
Monocytes accounted for up to $25 \%$ of leukocytes (WBC) during active replication. As a rule, the decrease in CD14 expression preceded the decrease in HLA-DR expression (most of the CD14low showed HLA-DR) (Figure 4).

Generally, large dispersion of monocytosis values was observed (especially in the low viral load) and significant increase of viral load corresponded with low gradient of increase of monocytes count (Figure 1). Therefore, we analyzed the monocyte and viremia fluctuation in several patients with various CMV disease course and outcome (Figure 5). However, in our observation CD14+HLA-DR-negative monocytes remained marginal and sometimes not observed, the correlation with CMV-viremia level was $\mathrm{r}^{2}=0.4835$, contrary to CD14-DR- and overall DR-negative monocyte with $\mathrm{r}^{2}=-0.1468$ and -0.1264 , respectively. Only in individual situations a higher level of CD14+ DR(-) over CD14low was observed, as a hallmark of recovery, i.e. during the period of exponential decrease of viremia, when a significant decrease in CD14low monocytes was observed (Figure 5 B).

Changes in CD14 expression were significantly greater than those of HLA-DR. The decrease in CD14 was in line with the increase in viral load, except when there was a rapid recovery period without relapse (Figure 5a). However, the rapid increase of CD14low/CD14monocytes was observed in patients with fatal outcome (Figure 5c). 


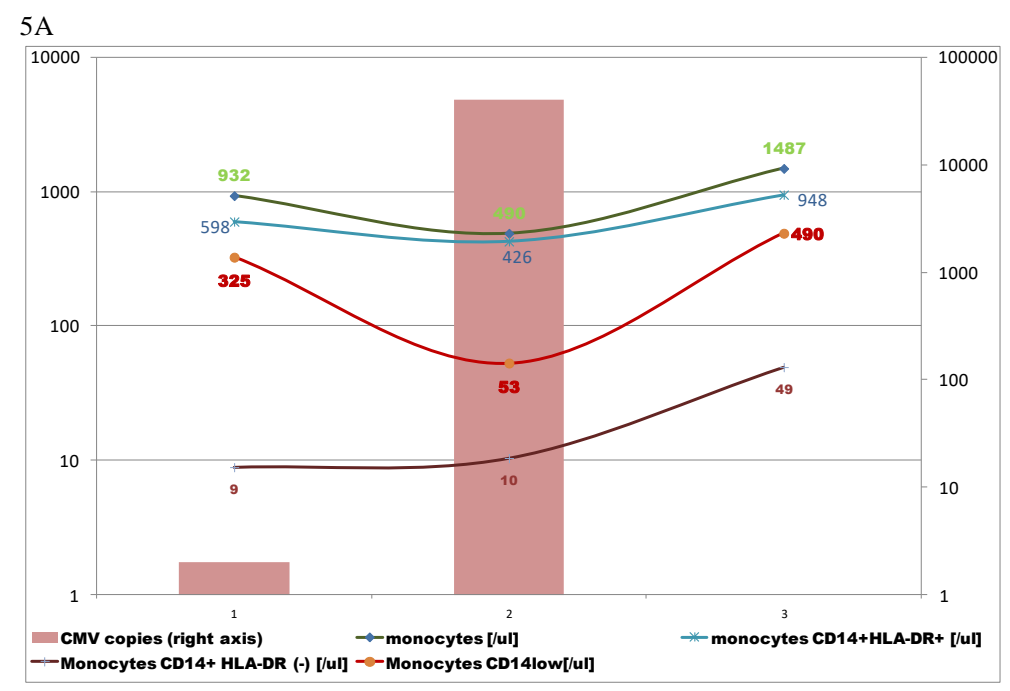

$5 \mathrm{~B}$

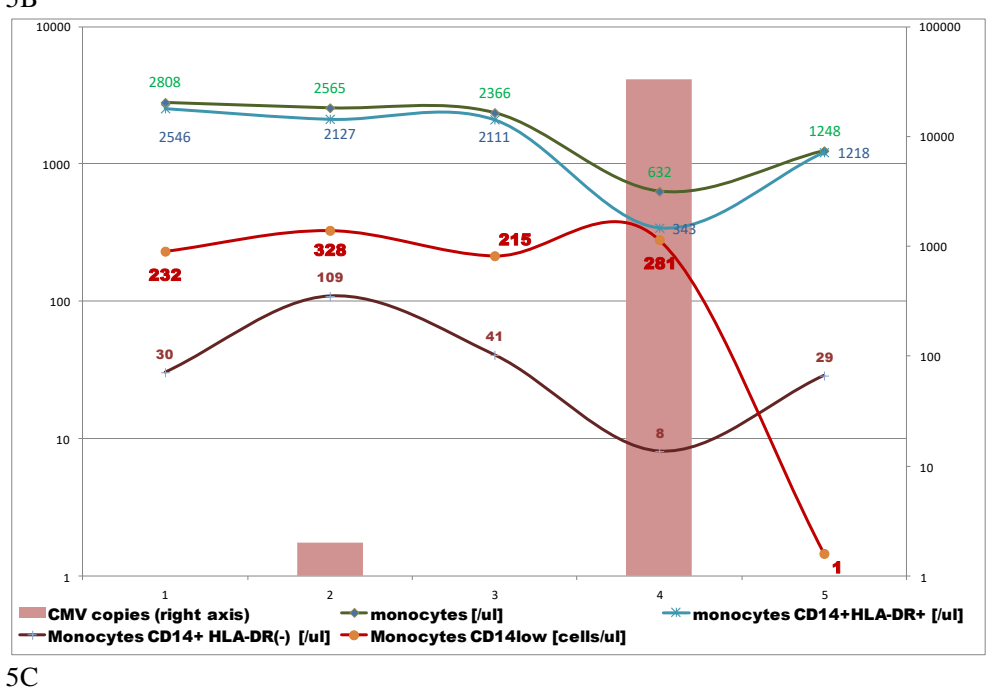

$5 \mathrm{C}$

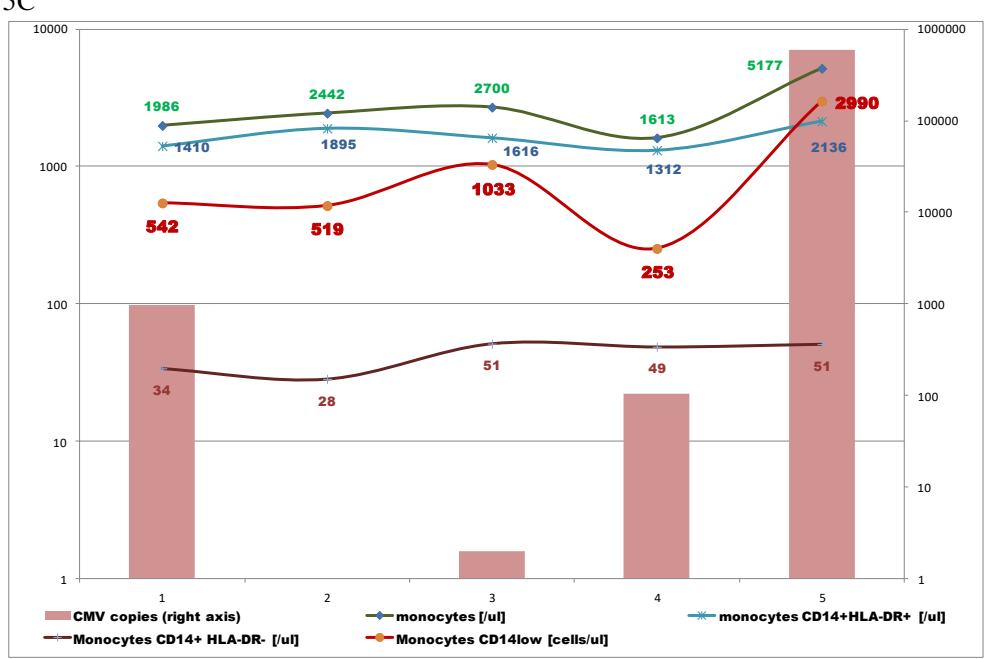

Fig. 5. Relationship between CMV-DNA and monocyte count and subpopulation .

The course of CMV disease is presented in the timeline until viremia withdrawal or fatal outcome.

Representative patient plots of each group are shown in 3 clinical situations and outcome:

5 A symptomatic infection with rapid latency, exponent $x=4,77$

5B oligosymptomatic infection with rapid relapse with intensive symptoms of CMV mononucleosis and good outcome $x=4,52$ $5 \mathrm{C}$ symptomatic primary infection with temporary viremia disappearance, prolonged intensive mononucleosis and relapse

with fatal outcome The exponential growth of viremia was the highest $x=5,77$

Noteworthy, initial low CMV viremia level was not tantamount to latency.

CMV copy numbers on 100,000 nucleated cells of the whole blood were expressed as the box on the right axis. 


\subsection{Potential role of steroids}

It is noteworthy that relative monocytosis is also described in minor viral infections (during the prodrome) and chronic corticosteroid therapy, but without respect to the dose of steroids and monocyte count [13].

Patients received high dose of steroids to develop higher level of CD14low monocytes than patients who received no or low dose (Figure 6). Furthermore, median value of CD14low were near comparable or higher than across the study (302/ $\mu$ l Figure $4 a)$ i.e. 312 or $542 / \mu$ l when patients received dose equivalent to $10 \mathrm{mg}$ or $20 \mathrm{mg}$ of dexamethasone, respectively (Figure 6). Moreover, the observed changes affected the absolute value, therefore the amount of lymphopenia did not result in an increase in monocytosis.

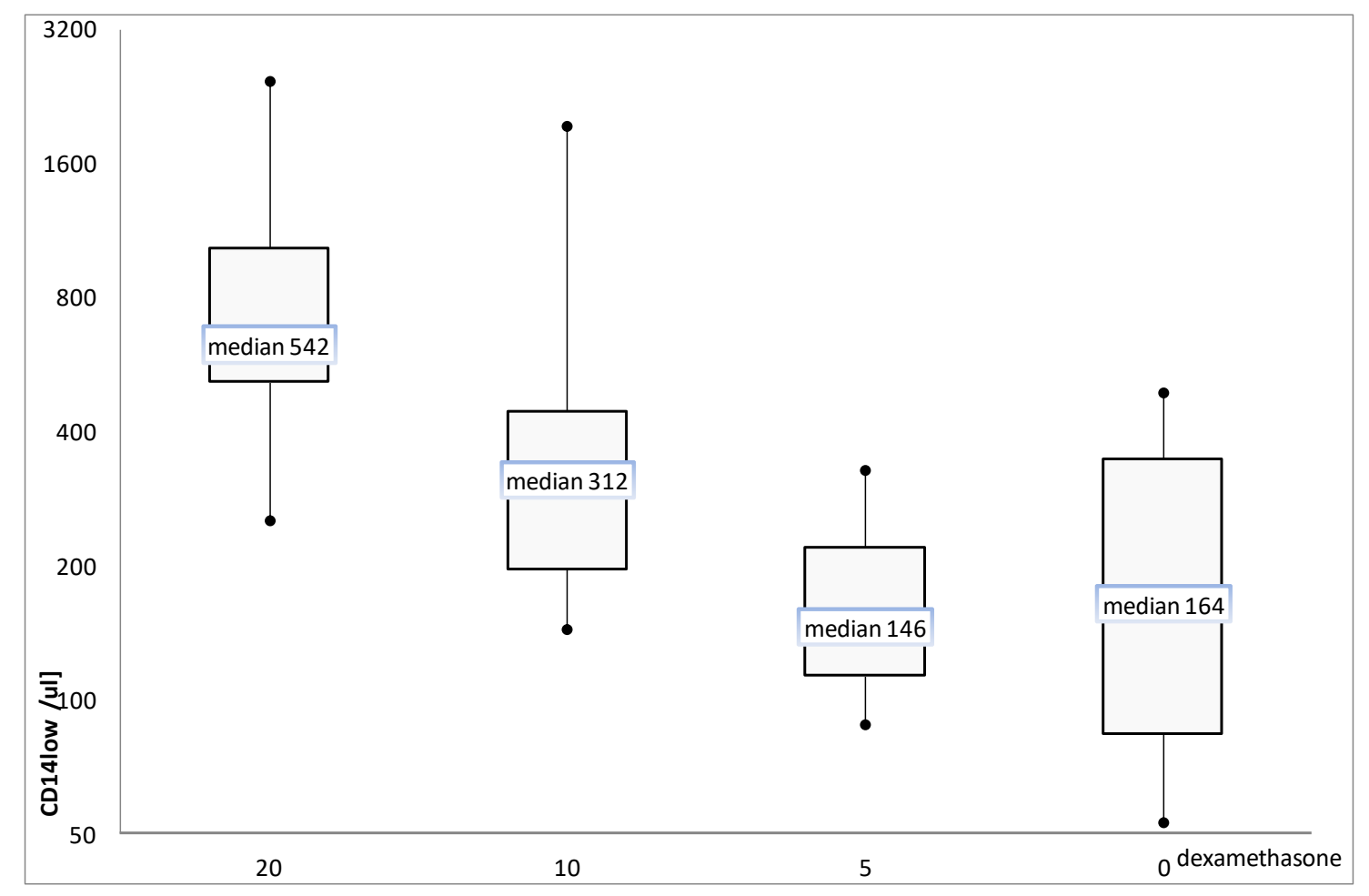

Figure 6. Various CD14low monocyte levels under the influence of ongoing glucocorticoid therapy.

For comparison CD14low was presented in control CMV+ patients that had never been treated with steroids. The dose of steroids was presented as an equivalent dose of dexamethasone that form a geometric sequence $\left(\operatorname{ar}^{\mathrm{n}}\right)$ with common ratio $\mathrm{r}=2$ and start term $\mathrm{a}=5$. 


\section{Discussion}

\subsection{Monocvte quantitation, description with different methods: influence on the basic characteristics of cells}

After hematopoiesis from myelomonocytic stem cells in bone marrow, monocytes move into the blood where they circulate with a half-life of 1-3 days [13]. Classical monocytes are the cells known to hematologists for a century as monocytes on the basis of the structure. Result of morphology-based examination is a leading parameter in clinical situation, especially in critically ill patients, when more specific enumeration and analysis by flow cytometry is too long for medical decision and preemptive therapy. Automatic analysis in the case of severe leucopenia (e.g. WBC $<100$ cells, monocyte count $<10$ ) is problematic, the analysis requires examination of a larger blood volume. In our observation the entire dataset from comparison presented in Figure 2 and 3, monocyte count analyzed simultaneously on hematology analyzer, flow cytometry (with CD45-gating or CD14+) and manual differential counts showed a high correlation coefficient (Table 1). Although CD14 expression is similar in fresh blood and Ficoll-isolated monocytes, the enumeration with cytometric methods using different standard beads is laborious and time-consuming [16]. In our observation monocytosis was observed regardless of the used technique (Figure 2) and may be the hallmark of active replication. In the clinical setting, the morphological definition will remain the standard because, despite numerous studies, there are no unambiguous, accepted cytometric norms. In most laboratories the monocyte count is calculated in flow cytometry after gating as the product of cells stained with CD14 and WBC (noteworthy, from hematology analyzer).

Several methods could be used for quantitation of monocytes but cell counting evolved a somewhat non-repeatable model [17,18]. Therefore, standard approach in clinical manual analysis and hematology analyzers relies on physical properties of these cells including light scatter. Our preliminary analysis with forward and side scatter (FSC/SSC) examination of monocytes in our patients was not useful, because it did not discriminate well between leukemic blasts, lymphocytes and monocytes. A large proportion of the studied patients were with lymphomas as well as with adaptive immune response disorders (Table 2). Our observation of good accuracy of CD45/SSC gating and other methods (Figure 1,2) corresponds with other studies on hematologic diseases. As described by Lacombe and coworkers, they discriminate well between monocyte, leukemic blasts, that are CD45low [19]. Although correlation between flow hematology analyzers and manual MGG smears is high, in scientific and experimental situation flow cytometry has been proposed as the 
reference method for the detection of various cells in blood, for instance monocytes and dendritic cells [20].The unexpected difference between CD14-positive cells in flow cytometry and monocytosis in hematologic analyzer were the first reason for further analysis in our study (Figure 2,3). All these cytometric studies and experimental research were based on Ficoll-based separation, single cell type identification with the use of specific antibody combination. It is not suitable for full differential count. The three candidate protocols were used for comparison and none of them used CD14, but CD16, HLA-DR and CD45 [18]. In our analysis direct (by hematology analyzer) and based on CD45 monocyte counting were used with high accuracy. We observed higher correlation and efficiency than in comparative study of several hematology analyzers [21]. In the study neutrophils and eosinophils also showed very good correlations, whereas lymphocytes and monocytes correlated fairly. Interestingly, in comparison with reference manual cell counts two of three protocols showed high (>0.9) correlation coefficient. The U.S. protocol showed intermediate correlation and low accuracy (CD33 and CD64 were used) [18]. Furthermore, in Ficoll-isolation procedure and many experimental studies, neutrophils for their part are found at trace and contaminating amounts in most PBMC preparations [22].

Our observation corresponds with Grimaldi's report, where hematology analyzer sometimes showed slightly higher monocyte counts than the manual (MGG-based) or CD14-based cytometric method [23]. Such analysis by itself may have slightly underestimated monocyte numbers, since only CD14 and not CD64, DR or CD33/side scatter was used for the detection of monocytes. Monocytes represent a much more heterogeneous population, and the use of only one cytometric marker without additional gating leads to inaccurate conclusions. (for technical aspects see a comparative study of several hematology analyzers) [23]. Interestingly, in our observation regression lines were nearly parallel and their slope coefficients were 0.8068 and 0.876 (Figure 3). All slope coefficients were below 1. Therefore, the difference between monocytes in the morphological and CD14-based assays in the acute phase of replication was almost a constant value close to 250 cells $/ \mu 1$ (i.e. $244.71=219.09+25.62-$ Figure 3). It corresponds with nearly the same difference in minimum, maximum and median value observed in the statistical analysis (Figure 4). One explanation is the fact that monocytes are CMV Trojan horse. Monocytes together with $\mathrm{CD} 34^{+}$progenitor cells were found in naturally infected cells [24]. Alternatively, the increasing monocyte count may be the result of half-life greater than 3 days and reduced recruitment into tissue macrophages or mobilization of peripheral pool (see below) [25]. 


\subsection{Clinical manifestation: significant monocvtosis under the influence of CMV replication, monocyte subtype}

CMV is a very immunoreactive virus. The range of CMV infection is wide, from CMV reactivation, presenting mainly as asymptomatic viremia to CMV end-organ diseases, such as esophagitis, gastroenteritis, hepatitis, retinitis, pneumonia, and encephalitis, but without strict laboratory criteria [6].

Bone marrow is the primary target of CMV infection and myelomonocytic stem cell precursors produce a constant number of monocytes from which the stable fraction is infected [26]. Thus, such strategy would allow the virus to escape from immunosurveillance and would increase the chances for lifelong latent infection and latent CMV down-regulates major histocompatibility complex [27]. Another study indicates increased expression of DQ on myeloid progenitor cell [28]. In the latent phase of infection, CMV activates a different set of genes [28]. However, our observation shows in active phase the infectious process and significant CMV replication in blood compartment, therefore downregulation of HLA-DR plays a minor role (Figure 3, and 1 with almost horizontal regression lines for DRmonocyte). Blood is the environment where monocytes contact with lymphocytes much more often. Moreover, lymphocytes and blood monocytes may be cells that left the lymphatic organs and entered the blood via the lymphatic system. Interestingly, CD14-negative bonemarrow monocyte pool was observed previously [29]. Although high dispersion of results was observed, correlation with viremia and slope of the regression line was similar for CD14low and total monocyte counts (Figure 1). Therefore, high monocyte count largely derived from CD14low level. Moreover, in clinical situation, the parallel course of the absolute number of monocytes and CD14low curves was observed especially in two extreme situations: with good and fatal outcome (Figure 5A and C, respectively).

The cause of monocytosis is primarily hematologic or infectious disease, but cytomegalovirus disease has not been described [13]. However, lymphocytosis with atypical lymphocytes is a well-known symptom in immunocompetent patients, but most of patients with active CMV replication are lymphopenic under the influence of NHL, HSCT or primary immunodeficiency (Table 2) [9,11]. High (up to $25 \%$ of WBC) monocyte percentage corresponds with such situation (Figure 4B). Therefore, the clinical description and sign of CMV disease is still an open issue [3]. The strong correlation between the number of monocytes and the viremia level indicates that high monocytosis (ranging between 1337 and 1723 depending on the method) may be a sign of cytomegalovirus disease and CMV replication (Figure 2). 
On the contrary positive results and high viremia level (i.e. about 1000 copies observed in whole group with median value 950 (Table 2) per se does not coincide with monocytosis as much as how during exponential growth (Figure 2). Moreover median value of monocyte level in whole group was comparable with healthy subjects with latent form of infectious process i.e. 362 and 400 respectively (Figure 2).

Specific immune response is crucial for CMV disease control, in part by surface IgG receptors such as CD16 and CD64) [11,30]. In our observation most of CD14low monocytes express HLA-DR (Figure 4), but it is not a rule. In another study intermediate (CD14+CD16+) monocytes exhibited an increased phagocytic activity, higher expression of CD11b and TLR4 as well as a decreased antigen presentation in comparison with CD14low non-classical monocytes [31]. Therefore, CD14low monocytes, observed here under the influence of CMV, may prompt via antigen presentation - adaptive, contrary to inhibition of innate immunity, especially tissue homing (by CD11/CD18), lipopolysaccharide (LPS)derived TNF $\alpha$ production [1].

HLA-DR-negative CD14+ monocytes are sporadically observed (median 31 cells/ $\mu \mathrm{l} ;<5 \%$ Figure 4), but usually patients develop primary or secondary adaptive immunity disturbances together with low CD14 (median CD14lowDR-79 cells/ $\mu 1$ as presented in Figure 4).

Explanation of the phenomenon may be concomitant lymphopenia and lack of $\mathrm{T}$ cell and monocyte interaction, for example by lymphocyte-derived Interferon gamma (IFN $\gamma$ ) secretion under the influence of antigen presentation (see below) [12]. High count of CD14low and their increasing number to values similar to CD14+ DR+ were the hallmark in patients with severe course, especially with fatal outcome (Figure 5 C). Furthermore, the viremia level showed higher correlation with CD14low than CD14+ classical monocyte level $\mathrm{r}^{2}=0.3501$ and 0.2140, respectively. Therefore, in our situation, decreased HLA-DR expression level was not a simple indicator of immunosuppression, contrary to a multicenter study [32]. Decreased expression of HLA-DR on human monocytes during sepsis was described by Lekkou et al., but methodological details showed that the analysis was performed only on CD14+ monocytes [33]. In other words, the decreased expression of HLA-DR on "monocytes" is not strict, because in most studies non-classical monocytes (CD14low) were not analyzed. Therefore, analysis of monocytes requires special attention because the frequency of some of these cells can be very low and misinterpreted as non-monocytes [14,16,20,34]. Transcriptomic analyses demonstrated that CD14low human monocytes were the counterparts of LY6Clow non-classical mouse monocytes, but it is not clear exactly which human cells are monocyte origin [35]. Classical monocytes are cells known on the basis of the 
structure, whereas the somewhat smaller, non-classical monocytes, which account for a small pool only, were described just 20 years ago [14,25]. Monocytes in blood have some typical morphological features (irregular shape of the cell and its nucleus, high cytoplasm-to-nucleus ratio, light blue cytoplasm in MGG staining). Last finding of Tak and coworkers, with MGG stained cytospin preparations, showed an increasingly mature phenotype from CD14+ to CD14low non-classical monocytes as characterized by a more neutrophilic cytoplasm and increasingly dendritic appearance [25]. Here, CD14low denotes an expression level that is similar to that of neutrophils. This approach indicates that in some situations, as observed here, during CMV infection, the more abundant neutrophils, despite the lower number of lipopolysaccharide receptors, are the first line in response to Gram-negative bacteria. It is in compliance with the proposed nomenclature, that non-classical CD14+ 10-fold above the isotype control and classical CD14 ${ }^{++}$is $\sim 100$-fold above the isotype control [34].

Most of studies analyzed intermediate and non-classical monocytes as a single CD16-positive subset [36]. Intermediate monocytes are transient monocyte population during differentiation between classical (CD14+CD16-) and non-classical (CD14lowCD16+ subtype) that express both surface markers $[14,25,34,35]$. The HLA-DR expression is higher in intermediate monocytes, but may be expressed on activated lymphocytes or dendritic cells and after FSC/SSC gating CD14negativeHLA-DR+ monocytes may be indistinguishable from activated lymphocytes that show higher volume [37, 38]. It corresponds with our preliminary research with not-useful FSC/SSC gating for monocyte analysis. Interestingly, the inclusion of pan-monocyte markers CD86 and major histocompatibility complex II in gating strategy (either with or without the inclusion of cells in the lymphocyte gate) resulted in a slightly higher number of monocytes, but their use did not result in significant differences in monocyte parameters [18]. The same phenomenon was presented here, when hematology analyzer or CD45/SSC gating resulted in higher number of monocytes than simple CD14based gating.

\subsection{CD14 role in CMV disease manifestation}

CD14 together with toll-like receptors (TLR) are a well-known PRR for LPS of Grammnegative bacteria. In addition, CD14 acts as a co-receptor for TLR2:TLR6 heterodimer in response to diacylated lipopeptides and for TLR2:TLR1 heterodimer in response to triacylated lipopeptides [39]. Recent finding has shown that the role of CD14 is more universal, CD14/TLR complex contributes to the detection of CMV [40]. Envelope glycoprotein $\mathrm{B}(\mathrm{gB})$ and $\mathrm{H}(\mathrm{gH})$, display determinants recognized by TLR2 [41]. CD14low 
monocytes are weak phagocytes and they do not produce reactive oxygen or cytokines in response to cell-surface Toll-like receptors [20]. It explains why most patients with CMV replication develop later bacterial coinfection or superinfection. In our practice the CMV reactivation is not per se the cause of fatal outcome. Therefore, CMV replication, outcome and overall survival are more complex (Figure 5). Furthermore, the CD14/TLR complex is extracellular sensor, therefore in latent form is of minor significance. The significant increase of CD14low in count and percentage was observed, but the difference between CD14low non-classical and active CD14+ classical monocytes is less visible in percentage (Figure 4B). Unfortunately, the observed here decrease of CD14 during exponential replication, when virions and entry-mediating $\mathrm{gB}$ and $\mathrm{gH}$ are available for TLR2, the innate immune response is not activated due to the lack of CD14-coreceptor (Figure 7). It explains our previous observation of crucial adaptive immune response, especially IgG in CMV in primary immune response against CMV reactivation during infancy or after HSCT [11]. Moreover, high expression of HLADR on CD14+ as well as CD14low monocytes (Figure 4) and significant absolute monocytosis prompt $\mathrm{T}$ and subsequent $\mathrm{B}$ cell activation, therefore adaptive immune response (Figure 7). However, the adaptive immune response is per se highly specific and vigorous specific immune response to $\mathrm{CMV}$, in part due to changes in monocytes (Figure 7), does not correspond with immune response to other pathogens [42]. Therefore such immune response switching may be the cause of secondary coinfections and CMV latency.

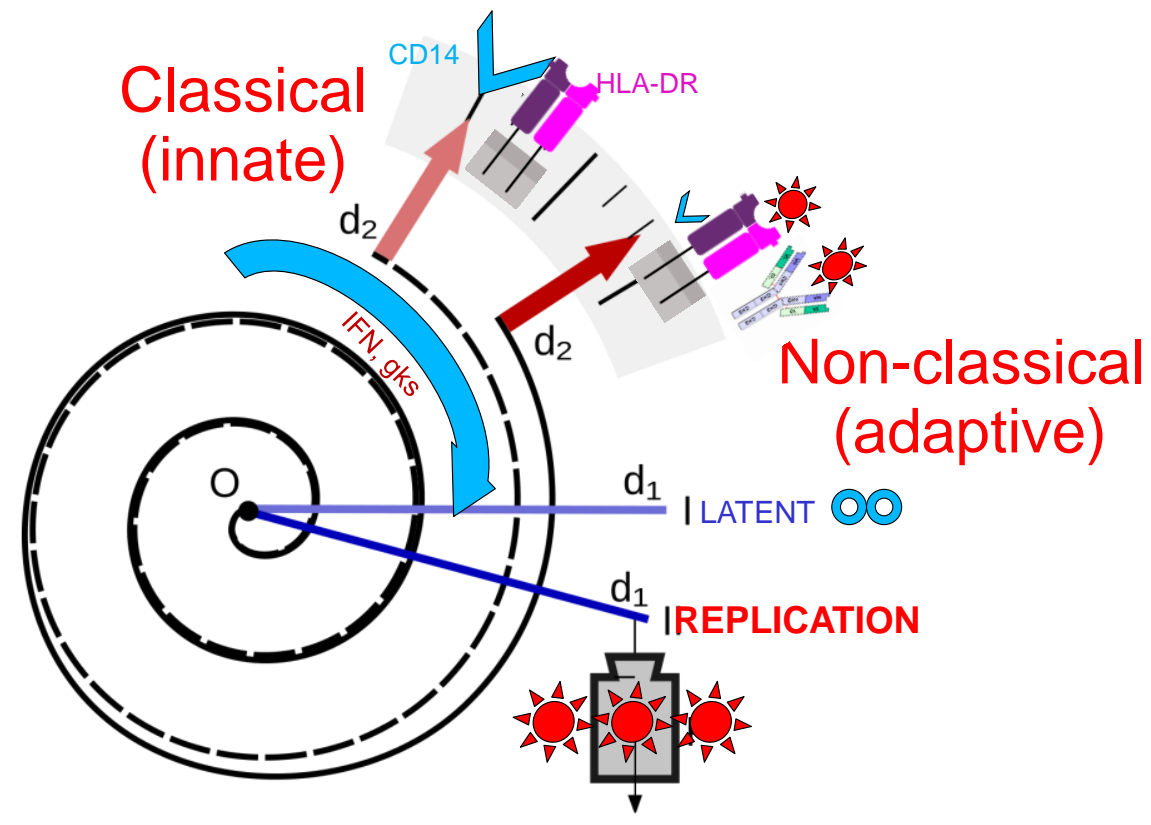

Figure 7 Balance of Blood Monocyte subtype under the influence of CMV and glucocorticoids. In latent phase many genes of innate immune response were upregulated in myeloid precursors by $\mathrm{CMV}$ as described by Slobedman et al., but under the influence of active replication CD14 decrease and further machinery of monocyte differentiation progresses. Noteworthy, in several patients the main target of CMV replication was 
monocytes, as a results of immunodeficiency and lymphopenia (Figure 4b). It prompts significant increase of monocyte level with non-classical signature. The intracellular localization of virus particle in latency causes lack of CD14/TLR2 $\leftrightarrow \mathrm{CMV}$ interaction. However, in replicative phase, when the CMV envelope proteins $(\mathrm{gB}, \mathrm{gH})$ are available, the non-classical signature (CD14lowCD16+) monocytes are activated by adaptive mechanism - IgG and surface receptor (CD16). High expression of HLA-DR is the second pathway of adaptive immune response progress. Non-classical monocytes are not activated by innate (TLR2) pathway due to low expression of CD14coreceptor.

Decrease of CD14 on lung macrophages was observed previously, but the research was rather experimental: the infectious process was developed in vitro after incubation of high titer of CMV strain AD 169 [43]. Our first clinical observation is: the same process developed in blood, during exponential growth of viremia and usually lymphopenia with significant increase of CD14low monocyte count and percentage (Figure 1,4 and 5).

In the timeline the CD14 decreased parallely with the increase in viral load and preceded significantly HLA-DR decrease (see timeline in Figure 5 b, c), except when there was a rapid recovery period (Figure 5 a). The same rule is not observed when we look at viremia in stationary phase in the same patient and all patients with positive CMV results i.e. patients with $\geq 100 \mathrm{CMV}$ copies $/ 10^{5}$ cells (Fig. 2) [12]. The reason may be the changes in the number and distribution of leukocytes due to the applied treatment for example myelotoxic drugs, such as ganciclovir (Table 2). It is worth noting that in secondary immunodeficiency patients in the valgancyclovir arm experienced a statistically significant early and prolonged decrease in their monocyte counts followed by a transient increase during the post-treatment and an increase in absolute neutrophil counts [44]. Furthermore, in our observation among HLA-DRnegative monocytes CD14low are much more abundant than CD14+ during active CMV replication (Figure 4).

\subsubsection{Potential cause of CD14 decrease}

Recent finding shows that membrane receptor recognizes CMV envelope protein, leading to innate immune activation and pattern recognition receptors. Toll-like receptor 2 (TLR2) and CD14 recognize CMV virions and trigger inflammatory cytokine production as described by Compton et al. [40]. Therefore, CMV and CD14 interaction may be a simple cause of competition between CMV and staining with anti-CD14 MoAb. In such situation the above mentioned difference between hematology analyzer or CD45 gating and CD14-positive cells should depend on viremia level. Unfortunately, it was practically constant (Figure 3). In most patients, the highest viral load did not coincide with the peak of CD14low. Only in patient with fatal outcome the parallel increase was observed in the end stage of the disease when the CMV load was about 590000 per 100000 nucleated cells (Figure 5C). Furthermore, the 
CD14low non-classical monocyte levels showed low correlation low and linear increasing tendency with exponential growth of CMV viremia (Figure 1).

\subsubsection{Monocvte differentiation}

When we look at the expression of individual molecules during active CMV replication with absolute and relative monocytosis, the monocyte populations form the following series: classical activated monocytes (CD14+HLA-DR+)> non-classical activated monocytes (CD14lowHLA-DR+) >> remaining non-classical (CD14lowHLA-DR(-) $>$ and residual classical inactive pool (CD14+HLA-DR(-) (Figure 4 and 5b,c) with various tendency: increasing for classical and CD14low HLA-DR+, decreasing for CD14lowHLA-DR(-) (Figure 1). It corresponds with the recent observation and model of gradually changing expression patterns, combined with their consecutive monocyte repopulation kinetics. Monocytes were shown to differentiate from classical via intermediate to non-classical monocytes as described by Tak et al. [25]. It was suggested that the last differentiation step takes place outside the circulation. Furthermore, the kinetics was very similar for both: high or low rate of cell division of precursors in the bone marrow. It indicates that monocytosis observed here under the influence of CMV may depend on monocyte kinetics rather than on marrow overproduction. Furthermore, Tak et al. model shows that blood residence is high for non-classical and low - for intermediate monocytes. In healthy individuals post-mitotic pool in blood contains about 500/ $\mu 1$ classical and 200/ $\mu 1$ CD14low non-classical monocytes in contrast to our observation in CMV patients, where these numbers were 2-3 times higher (Figure 1,4a). In the percentage formula, they constituted $90 \%$ and $5-10 \%$ of monocytes in healthy individuals [25] and in our observation $79 \%$ and $19 \%$ monocytes, respectively. The significant increase in count and percentage was observed, but the difference between CD14low non-classical and active CD14+ classical monocytes is less visible in the percentage (Figure 4B). Interestingly, two explanations were proposed - direct differentiation of classical monocytes without intermediate step or non-circulating pools of CD14low with differentiation step outside the blood [25]. Probably blood monocytes may be cells that left the blood to lymph node, and then returned into the circulatory system by right lymphatic or thoracic duct. It corresponds with our patients presentation: temporary and significant decrease of CD14low monocytes in blood (down to a value below the baseline) was observed under the influence of CMV replication (Figure 5). The same developmental relationship between these cells (from classical to non-classical) was observed in another study: during the course of an infection or with macrophage colony-stimulating factor (M-CSF) + IFN $\gamma$ 
treatment [45]. In such circumstances an increase of the non-classical monocytes was described: CD14 was expressed by only 36\% of the cells. Significant CMV-induced IFN $\gamma$ release (with higher level than post-mitogenic) was observed in our previous study during significant CMV-reactivation and T cell lymphocytopenia [11]. Taken together these results indicate that IFN $\gamma$, that may be produced by both $\mathrm{T}$ and Nk cells, is the key factor for monocyte equilibrium: between innate and adaptive immunity (Figure 7). Such two faces of monocytes in our study are seen in decreased expression of CD14 and the presence of HLADR on many cells (Figure 4 and Figure 5).

The third explanation of CD14low monocytes increase may be in part the influence of longterm immunosuppressive steroids. So we compared CD14low monocytes level in 8 patients (selected from the first group of 16 patients) in latent phase with different doses of steroids converted to dexamethasone (Table 2) [46]. The effects of steroids and CMV replication were therefore observed separately (see Material and methods). Although patients received high dose glucocorticoids in various periods and for various indications, the virus replication was not observed, but low median monocyte level was observed, contrary to active replication period ( Table 2). Then, we compared the geometric progression of dexamethasone dose with the values of CD14low monocytes per $1 \mu \mathrm{l}$ : it formed also geometric sequence with common ratio about 2. The rule was observed at highest doses (i.e. 10-20mg). 10mg of dexamethasone gave the two-fold higher CD14low content than observed in the control group of CMVpositive patients, never treated with steroids (Figure 6). However, steroid equivalent to 10mg of dexamethasone did not produce any significant effect as compared with median value of CD14low across the study $(302 / \mu 1)$. It also indicates that positive CMV results (in our Center $>100$ copies [12]) is not synonymous with cytomegalovirus disease and median viremia about 1000 may be observed without existing progress of CMV replication (Table 2, Figure 2) without significant monocytosis, as seen in the regression analysis and the large dispersion of the results (Figure 1). In addition, $5 \mathrm{mg}$ of dexamethasone showed no effect.

It is the first clinical presentation of dose-dependent effect of steroids on CD14 expression in patients with latent CMV. It is not to be underestimated in the therapeutic application of dexamethasone in hemato-oncological chemotherapy.

Noteworthy, in our situation such clinical conditions resemble experimental ones: no wash procedures or cell separation were used and till laboratory analysis "native environment" and steroid influence were preserved. As described previously, dexamethasone downregulated cellular and surface levels of CD14 and blocked the release of soluble CD14, but noteworthy without altering gene transcription [47]. Furthermore, the study overcame the difficulty of 
nomenclature and monocyte definition, although based on a cell line (THP-1 monocyte/macrophage cells) and in vitro culture. The monocyte count was 250/ $\mu 1$ (usually observed in clinical setting; Figure 2) but monocyte exposition on 0.01-1 $\mu \mathrm{M}$ dexamethasone was also high (i.e. $0.0039-0.39 \mathrm{mg} / \mathrm{l}$ ) and comparable with presented here. CD14 binds electronegative low-density lipoprotein (LDL) and mediates the cytokine release induced by LDL [48]. Furthermore, as presented here, glucocorticosteroids - known pro-atherogenic and pro-diabetic agents - may interfere with atherogenesis by modification of CD14 expression in dose-dependent manner.

Our study has many limitations, such as too few patients and a simple technique. A very restrictive criterion such as the exponential increase in viral load is one of the basic ones, although it brings us closer to in vitro research. It is difficult to plan a clinical trial in which steroids are directly tested during CMV infection (effects of steroids and CMV were observed separately). On the other hand, their influence not only on lymphocytes but also on monocytes seems to be noticeable (Figure 6). Monocytes recognize CMV in a nonspecific manner with the participation of CD14 but also specifically through IgG and CD16, expressed on nonclassical CD14low subtype. In this light, the balance between classical and non-classical monocytes seems crucial (Figure 7).

Patients infected with CMV are usually with multiple co-morbidities with various therapeutic regimens, usually with steroids. On the other hand, the growing number of experimental studies does not coincide with the progress in diagnostics and symptomatology. Primary immune response may be observed during secondary infection and vice versa [11]. Nomenclature problems make it difficult to compare the results $[6,8,11]$. Also the results obtained in different sections of our laboratory are sometimes divergent ( e.g. delivered by experienced hematologists and immune-cytometric analysis). Therefore, the study used the simplest possible techniques, such as CD45 / SSC gating, single molecules and publicly available clinical methods. A better laboratory definition of CMV replication and disease, such as proposed here, the exponential growth of viremia, could facilitate further progress in the clinical environment.

\section{Material and Methods}

\subsection{Material}

After approval of the local ethics committee (KB-52/2010), the active CMV disease records were reviewed and analyzed. Cases were identified either at the time of hospital admission or 
while analyzing the medical record at follow-up appointment at the outpatient clinic for diagnosis of immunodeficiency (primary or secondary), as a control post HSCT or residual disease monitoring after therapeutic interventions in leukemia/lymphoma. The material for this single-center study was derived from a group of $160 \mathrm{CMV}$-positive patients with positive CMV viremia whose peripheral blood samples had been analyzed (see below). Sequential analysis of EBV, HHV6 and CMV viremia, WBC, peripheral blood monocytes, C-reactive protein, bacterial or fungal culture were performed. It is recommended that studies report separately cases where CMV disease is found with or without co-pathogens with details given on the co-pathogens [6] (Table 2). 


\begin{tabular}{|c|c|c|c|}
\hline Characteristics & \multicolumn{3}{|l|}{$\begin{array}{l}\text { All participants } \\
(\mathbf{n}=160)\end{array}$} \\
\hline Median age (range) & \multicolumn{3}{|l|}{$45(20-65)$} \\
\hline Sex; Man $(\%)$ & \multicolumn{3}{|l|}{$83(52 \%)$} \\
\hline $\begin{array}{l}\text { Mean viral load [copies per } 100000 \\
\text { nucleated cells] }\end{array}$ & \multicolumn{3}{|c|}{$950(100-590000)$} \\
\hline \multicolumn{4}{|l|}{ Underlying disease } \\
\hline -Primary immunodeficiency & \multicolumn{3}{|c|}{45} \\
\hline \multicolumn{4}{|l|}{-Secondary immunodeficiency } \\
\hline - $\quad$ after HSCT & \multicolumn{3}{|c|}{54} \\
\hline - lymphoproliferative disease & \multicolumn{3}{|c|}{48} \\
\hline - Cancer & \multicolumn{3}{|c|}{16} \\
\hline \multicolumn{4}{|l|}{ Exclusion criteria*: } \\
\hline Bacterial infection (Gramm-negative) & \multicolumn{3}{|c|}{$52(44)$} \\
\hline $\begin{array}{l}\text { C-reactive protein without known bacterial } \\
\text { specimen }\end{array}$ & \multicolumn{3}{|c|}{35} \\
\hline HHV6 & \multicolumn{3}{|c|}{5} \\
\hline EBV & \multicolumn{3}{|c|}{36} \\
\hline HSV $1 / 2$ & \multicolumn{3}{|c|}{8} \\
\hline $\mathrm{VZV}$ & \multicolumn{3}{|c|}{2} \\
\hline Fungal & \multicolumn{3}{|c|}{4} \\
\hline Tuberculosis or other mycobacteria & \multicolumn{3}{|c|}{2} \\
\hline Current therapy with myelotoxic drug** & \multicolumn{3}{|c|}{12} \\
\hline Lack of content or uncooperative patient & \multicolumn{3}{|c|}{3} \\
\hline After exclusion & $\begin{array}{c}\text { Normal } \\
\text { subjects } \\
(\mathbf{N}=\mathbf{2 0}) \\
\end{array}$ & $\begin{array}{l}\text { Included } \\
(\mathrm{N}=16)\end{array}$ & $\begin{array}{c}\text { Steroids } \\
\text { subgroup } \\
\quad(\mathbf{N}=8) \\
\end{array}$ \\
\hline Median age (range) & $48(20-72)$ & $38(25-60)$ & $40(30-55)$ \\
\hline Sex; Man (\%) & $10(50 \%)$ & $8(50 \%)$ & $3(38 \%)$ \\
\hline Mean Viral load & $\begin{array}{c}31 \\
(6-89) \\
\end{array}$ & $\begin{array}{c}5875,50 \\
(100-22293) \\
\end{array}$ & $\begin{array}{c}28 \\
(0-30) \\
\end{array}$ \\
\hline $\begin{array}{l}\text { Median monocyte level*** } \\
\text { (range) }\end{array}$ & $\begin{array}{c}400 \\
(155-680)\end{array}$ & $\begin{array}{c}1550 \\
(600-2400)\end{array}$ & $\begin{array}{c}238 \\
(53-2295)\end{array}$ \\
\hline
\end{tabular}

Table 2 Patients characteristics

A small group of patients was characterized with CMV disease with virus replication i.e. exponential growth of viremia. For comparison a group of normal subjects with active replication and CMV disease, with anti-CMV IgG and latent form of infection was presented. Steroid subgroup included eight patients, but during a period of time when the result of viremia was negative (less than 100 copies in two or more measurements) and the patients received steroids because of various indications.

*In several patients two or more exclusion criteria were frequently observed.

** isotretinoin, ganciclovir, metronidazole, macrolide, chloramphenicol or tetracycline

*** monocyte level in hematology analyzer examination 
Therefore, we had a small selection of patients and that is why we compared our data with simple experimental (in vitro) models where usually a single factor was analyzed. Due to the usual occurrence of coinfection most of patients were disqualified from the analysis: concomitant viral (especially by EBV and other herpesvirus), bacterial, fungal or rickettsial infections were excluded. The presence of CMV in the blood, together with symptoms and/or signs, is not sufficient for the definition of either proven or probable CMV disease at any other site [6]. As shown in figure 1 results about 10,000 virus copies were obtained sporadically.

For this reason the cytomegalovirus disease and CMV replication were controlled. Patients were included when exponential growth of CMV viremia was observed in 2 or more CMVDNA analyses (the values form a geometric progression). Exponential function of viremia with various bases (b) that are a positive real number higher than 1 and whole copy CMV number as a values of function $f(x)=b^{x}, \quad$ when $b>1 ; x>0$.

In our study we used the common logarithm i.e. with base 10 (that is $b=10$ ).

Therefore, only patients with short time of significant CMV replication and unquestionably active CMV infectious process were qualified with minimal influence of other pathogens. After the significant growth of viremia patients received standard gancyclovir or valgancyclovir therapy. Because contraindications in long-term glucocorticoids treatment are acute viral diseases (especially major viral infections) as well as severe primary immunodeficiency, it was impossible to observe patients with exponential increases in viral load and steroid use.

In further long-term observation in various periods and for various indications (GVHD, severe drug hypersensitivity reactions, allergic conditions, lymphoid interstitial pneumonia, cerebral edema), steroids were used (often in one patient several times, in different doses with at least a month break). Due to the inhibition of the immune response by steroids and the 
pleiotropic effect, the viral load was monitored before and during glucocorticoids therapy. Treatment with steroids was not initiated when patients were herpesvirus, fungal or other infectious diseases positive.

The effects of steroids were tested in the period of 2-3 weeks after the start of the therapeutic regimen.

The studies were compared with the control group of patients with good quality of life, not receiving ganciclovir or steroids, and having repeated CMV negative viremia tests (Table 2).

\subsection{Methods}

\subsubsection{Estimation of burden of CMV disease}

Quantitative CMV DNA analysis in peripheral blood cells was determined using real-time PCR with Light Cycler II (Roche, Mannheim, Germany) and expressed in numbers of CMV copies in 100,000 nucleated cells of the whole blood as described previously $[12,49]$. For strict evidence of viral multiplication, virus replication in our situation was defined as exponential growth of DNA-viremia. Obviously, their exponential increase makes the absolute number of CMV copies significantly depending on the timing, blood sampling and frequency of measurement, especially in patients after HSCT. It should be noted that last evidence suggests that the detection of virus, antigen, or DNA in blood does not mean that CMV is replicating in blood [6].

\subsubsection{Monocvte analvsis}

The whole blood analysis was performed to avoid possible biases through artificial stimulation of monocytes arising from preanalytical steps by mononuclear cell separation (Ficoll-density gradient centrifugation), sample cryopreservation [50]. To obtain higher precision and higher accuracy no wash procedures were used [51]. Such probes preserve the "native environment" including cytokine produced cells, hormones, but also administered drugs that were removed by washing in other models [52]. Forward and side scatter (FSC vs 
SSC) was used initially to remove contamination and non-cellular elements. Preliminary study consists of FSC/SSC analysis of monocyte population and comparison with other techniques. Further on, the leukocyte analysis was assessed in whole blood by flow cytometry using classical CD45 gating as described previously [12].

In brief, the panel of monoclonal antibodies (mAbs) for the detection of surface CD45, CD14 and HLA-DR was purchased from Becton-Dickinson (San Jose, CA). Cells were stained for $30 \mathrm{~min}$ at room temperature. Human monocyte population was selected from whole blood based on gating on live CD45+ (LCA leukocyte common antigen) expression and SSC [10,19]. The cells were analyzed with FACScalibur Flow Cytometer (Becton-Dickinson), using CellQuest software for data acquisition and the data were analyzed. Data were expressed as the \% nucleated cells (i.e. \% WBC) or cell count per $1 \mu 1$. CD14low monocytic cells were considered to be those selected by morphology and CD45 which showed granulocyte-like CD14 expression manner [20]. The isotype control and the specific antibody were derived from the same process.

For comparison monocyte counts were determined from the same samples using the hematology autoanalyzer. The Complete Blood Count (CBC) was performed with the Sysmex XN-2000 or ABC-Micros and compared to monocyte count on the basis of a primary CD45/SSC order to give numbers of gating procedure. May-Grünwald-Giemsa stain (MGG smears) was performed. Experienced hematologists carried out differential counts on MGG slides. It is currently a technique recommended by Clinical and Laboratory Standards Institute as means to generate a reference differential count in method comparisons [17,18]. Pearson coefficients were used for comparison between manual counting, cell counter counts and flow cytometry. 


\section{Conclusions}

CMV disease is inadequately defined, viral load cut-off levels have not yet been defined and the term "replication" is used to indicate evidence of viral multiplication, sometimes used instead of CMV infection [6]. Therefore, for strict definition exponential growth of CMV viremia in two or more CMV-DNA analyses may be a new approach ensuring greater compatibility with experimental (in vitro) studies. The significant increase and exponent of the viremia function may be a new indicator of CMV disease. Low copy value, i.e. lacking CMV copies in small blood sample or having <100 CMV copies / $10^{5}$ cells, does not mean latency (no virus replication) as presented in Figure 5. The morphology based approach will still remain the basis of medical interventions (blood smear may still be useful due to the availability and recent useful observation described by Tak and coworkers) [25]. Because of recent finding which showed an increasingly mature phenotype from CD14+ classical to CD14low non-classical monocytes (increasingly neutrophilic cytoplasm and dendritic appearance), the monocyte nomenclature, definition and counting in clinical studies are still an open issue (based on morphologic/cytometric phenotype or ontogeny) [34,35].

Until now, CMV-induced decreased expression of CD14 on cells of monocytic origin has been observed in vitro and our observation shows comparable clinical situation in human blood. However, significant CMV-induced monocytosis observed here has no counterpart in in vitro studies. In the era of increasing use of dexamethasone, as in the treatment of COVID19, the effect on CD14 expression and blocking further stages of non-specific monocyte activation with monokine release seem to be one of the key factors in the clinical setting. Although T or NK cell-produced interferon gamma (IFN $\gamma$ ) level and genotype is crucial during CMV reactivation, the influence on monocytes subsets should be studied further $[11,12]$. Further studies will allow to show whether the reduction in expression of adhesion molecules associated with CD14 decline is the main cause of monocytosis.

\section{Notes}

\section{Author Contributions}

P.Z. collected and analyzed data, wrote, reviewed, and revised the manuscript. A.G. at the Institute of Immunology provided funding for the work and read the manuscript.

\section{Funding}

The research was supported by and statutory activity of Hirszfeld Institute. 


\section{Acknowledgments}

The author wish to thank Dr. Dłubek D. for flow cytometry analysis as well as Dr. Jaskuła E. for PCR examination.

\section{Conflicts of Interest}

The author declares no conflict of interest.

Ethics approval and consent to participate

Not applicable: retrospective nature of the study.

Consent for publication

Written consent for publication of clinical details was obtained from the patient in accordance with the 5 Declaration of Helsinki.

\section{Abbreviations}

cytomegalovirus (CMV)

Complete Blood Count (CBC)

forward and side scatter (FSC/SSC)

human leukocyte antigen (HLA)

Interferon gamma (IFN $\gamma)$

leukocytes $=$ white blood cells $(\mathrm{WBC})$

leukocyte common antigen (LCA)

macrophage colony-stimulating factor (M-CSF)

pathogen recognition receptors (PRRs)

toll-like receptors (TLR)

May-Grünwald-Giemsa stain (MGG smears)

\section{References}

1 Płóciennikowska A, Hromada-Judycka A, Borzęcka K, Kwiatkowska K. Co-operation of TLR4 and raft proteins in LPS-induced pro-inflammatory signaling. Cell Mol Life Sci. 2015;72(3):557-581. doi:10.1007/s00018-014-1762-5

2 Nguyen CC, Kamil JP. Pathogen at the Gates: Human Cytomegalovirus Entry and Cell Tropism. Viruses. 2018; 10: 704. doi:10.3390/v10120704

3 Horwitz CA, Henle W, Henle G, et al. Clinical and laboratory evaluation of cytomegalovirus-induced mononucleosis in previously healthy individuals. Report of 82 cases. Medicine (Baltimore). 1986; 65: 124-134. doi:10.1097/00005792-198603000-00005 4 Gugliesi F, Coscia A, Griffante G, et al. Where do we Stand after Decades of Studying Human Cytomegalovirus?. Microorganisms. 2020; 8: 685.

doi:10.3390/microorganisms8050685

5 Slobedman B, Mocarski ES. Quantitative analysis of latent human cytomegalovirus. $J$ Virol. 1999;73: 4806-4812. 
6 Ljungman P, Boeckh M, Hirsch HH, et al. Definitions of Cytomegalovirus Infection and Disease in Transplant Patients for Use in Clinical Trials. Clin Infect Dis. 2017;64: 87-91. doi:10.1093/cid/ciw668

7 Eddleston M, Peacock S, Juniper M,Warrell DA: Severe cytomegalovirus infection in immunocompetent patients. Clin Infect Dis 1997; 24 : 52-56

8 Ruef C. Primary CMV infections an important differential diagnosis in outpatients. Infection. 2003;31: 307. doi:10.1007/s15010-003-7503-6

9 Park KH, Ryu JH, Bae H, et al. Delayed NK Cell Reconstitution and Reduced NK Activity Increased the Risks of CMV Disease in Allogeneic-Hematopoietic Stem Cell Transplantation. Int J Mol Sci. 2020;21(10):3663. Published 2020 May 22. doi:10.3390/ijms21103663

${ }^{10}$ Ljungman P, Styczynski J, Einsele H. Viral Infections in ESH-EBMT Handbook on Haemopoietic Stem Cell Transplantation By J.Apperley, E. Carreras, E. Gluckman, T. Masszi Ed. 2012 p.281-290

11 Zdziarski P. CMV-Specific Immune Response-New Patients, New Insight: Central Role of Specific IgG during Infancy and Long-Lasting Immune Deficiency after Allogenic Stem Cell Transplantation. Int J Mol Sci. 2019;20: 271. doi:10.3390/ijms20020271

${ }^{12}$ Jaskula E, Dlubek D, Duda D, Bogunia-Kubik K, Mlynarczewska A, Lange A. Interferon gamma 13-CA-repeat homozygous genotype and a low proportion of CD4(+) lymphocytes are independent risk factors for cytomegalovirus reactivation with a high number of copies in hematopoietic stem cell transplantation recipients. Biol Blood Marrow Transplant.

2009;15(10):1296-1305. doi:10.1016/j.bbmt.2009.06.008

${ }^{13}$ Wallach's Interpretation of Diagnostic Tests Pathways to Arriving at a Clinical Diagnosis Tenth Edition Wolters Kluwer Philadelphia, 2015

${ }^{14}$ Ziegler-Heitbrock L. Monocyte subsets in man and other species. Cell Immunol. 2014;289(1-2):135-139. doi:10.1016/j.cellimm.2014.03.019

${ }^{15}$ Peters JH, Ruppert J, Gieseler RK, Najar HM, Xu H. Differentiation of human monocytes into CD14 negative accessory cells: do dendritic cells derive from the monocytic lineage? Pathobiology 1991;59:122-6.

${ }^{16}$ Antal-Szalmas P, Strijp JA, Weersink AJ, Verhoef J, Van Kessel KP. Quantitation of surface CD14 on human monocytes and neutrophils. J Leukoc Biol. 1997;61:721-728. doi:10.1002/jlb.61.6.721

17 Clinical and Laboratory Standards Institute. Reference leukocyte (WBC) differential count (proportional) and evaluation of instrument methods; Approved standard. 2007; 27.

18 Roussel M, Davis BH, Fest T, Wood BL; International Council for Standardization in Hematology (ICSH). Toward a reference method for leukocyte differential counts in blood: comparison of three flow cytometric candidate methods. Cytometry A. 2012; 81: 973-982. 19 Lacombe F, Durrieu F, Briais A, et al. Flow cytometry CD45 gating for immunophenotyping of acute myeloid leukemia. Leukemia. 1997; 11: 1878-1886. doi:10.1038/sj.leu.2400847

20 Ziegler-Heitbrock L. Blood Monocytes and Their Subsets: Established Features and Open Questions. Front Immunol. 2015;6:423. doi:10.3389/fimmu.2015.00423

21 Meintker L, Ringwald J, Rauh M, Krause SW. Comparison of automated differential blood cell counts from Abbott Sapphire, Siemens Advia 120, Beckman Coulter DxH 800, and Sysmex XE-2100 in normal and pathologic samples. Am J Clin Pathol. 2013;139: 641-650. doi:10.1309/AJCP7D8ECZRXGWCG 22. Jison ML, Munson PJ, Barb JJ, et al. Blood mononuclear cell gene expression profiles characterize the oxidant, hemolytic, and inflammatory stress of sickle cell disease. Blood. 2004;104(1):270-280. doi:10.1182/blood-2003-08-2760 
23. Grimaldi E, Carandente P, Scopacasa F, Romano MF, Pellegrino M, Bisogni R, De Caterina M. Evaluation of the monocyte counting by two automated haematology analysers compared with flow cytometry. Clin Lab Haematol. 2005 Apr;27(2):91-7. doi: 10.1111/j.1365-2257.2005.00676.x. PMID: 15784123.

24 Taylor-Wiedeman J, Sissons JG, Borysiewicz LK, Sinclair JH. Monocytes are a major site of persistence of human cytomegalovirus in peripheral blood mononuclear cells. J Gen Virol. 1991;72:2059-2064. doi:10.1099/0022-1317-72-9-2059

25 Tak T, Drylewicz J, Conemans L, de Boer RJ, Koenderman L, Borghans JAM, Tesselaar

K; Circulatory and maturation kinetics of human monocyte subsets in vivo. Blood 2017; 130: 1474-1477. doi: https://doi.org/10.1182/blood-2017-03-771261

26 Kondo K, Kaneshima H, Mocarski ES. Human cytomegalovirus latent infection of granulocyte-macrophage progenitors. Proc Natl Acad Sci U S A. 1994;91:11879-83.

27 Slobedman B, Mocarski ES, Arvin AM, Mellins ED, Abendroth A. Latent cytomegalovirus down-regulates major histocompatibility complex class II expression on myeloid progenitors. Blood. 2002;100:2867-2873. doi:10.1182/blood.V100.8.2867

${ }^{28}$ Slobedman B, Stern JL, Cunningham AL, Abendroth A, Abate DA, Mocarski ES. Impact of human cytomegalovirus latent infection on myeloid progenitor cell gene expression. $\mathrm{J}$ Virol. 2004 Apr;78(8):4054-62. doi: 10.1128/jvi.78.8.4054-4062.2004. PMID: 15047822; PMCID: PMC374258.

29 Cros J, Cagnard N, Woollard K, et al. Human CD14dim monocytes patrol and sense nucleic acids and viruses via TLR7 and TLR8 receptors. Immunity. 2010;33:375-386. doi:10.1016/j.immuni.2010.08.012

30 Ando T, Suzuki T, Ishiyama Y, et al. Impact of Cytomegalovirus Reactivation and Natural Killer Reconstitution on Outcomes after Allogeneic Hematopoietic Stem Cell

Transplantation: A Single-Center Analysis. Biol Blood Marrow Transplant. 2020; 26:171177. doi:10.1016/j.bbmt.2019.09.028

31 Skrzeczyńska-Moncznik J, Bzowska M, Loseke S, Grage-Griebenow E, Zembala M, Pryjma J. Peripheral blood CD14high CD16+ monocytes are main producers of IL-10. Scand J Immunol. 2008; 67: 152-159. doi:10.1111/j.1365-3083.2007.02051.x

32 Docke WD, Höflich C, Davis KA, et al. Monitoring temporary immunodepression by flow cytometric measurement of monocytic HLA-DR expression: a multicenter standardized study. Clin Chem. 2005;51: 2341-2347.

33 Lekkou A, Karakantza M, Mouzaki A, Kalfarentzos F, Gogos CA. Cytokine production and monocyte HLA-DR expression as predictors of outcome for patients with communityacquired severe infections. Clin Diagn Lab Immunol. 2004;11(1):161-167.

doi:10.1128/cdli.11.1.161-167.2004

34 Ziegler-Heitbrock L, Ancuta P, Crowe S, et al. Nomenclature of monocytes and dendritic cells in blood. Blood. 2010;116(16):e74-e80. doi:10.1182/blood-2010-02-258558

35 Guilliams M, Ginhoux F, Jakubzick C, et al. Dendritic cells, monocytes and macrophages: a unified nomenclature based on ontogeny. Nat Rev Immunol. 2014;14(8):571-578.

doi:10.1038/nri3712

36 Ong SM, Teng K, Newell E, et al. A Novel, Five-Marker Alternative to CD16-CD14 Gating to Identify the Three Human Monocyte Subsets. Front Immunol. 2019;10:1761. doi:10.3389/fimmu.2019.01761

37 Zawada AM, Rogacev KS, Rotter B, et al. SuperSAGE evidence for CD14++CD16+ monocytes as a third monocyte subset. Blood. 2011;118(12):e50-e61. doi:10.1182/blood2011-01-326827

38 Toapanta FR, Bernal PJ, Fresnay S, et al. Oral Wild-Type Salmonella Typhi Challenge Induces Activation of Circulating Monocytes and Dendritic Cells in Individuals Who Develop 
Typhoid Disease. Plos Neglected Tropical Diseases. 2015 Jun;9(6):e0003837. DOI: 10.1371/journal.pntd.0003837.

39.Triantafilou M., Gamper F.G., Haston R.M., Mouratis M.A., Morath S., Hartung T.,

Triantafilou K. Membrane sorting of toll-like receptor (TLR)-2/6 and TLR2/1 heterodimers at the cell surface determines heterotypic associations with CD36 and intracellular targetingJ. Biol. Chem. 2006; 281:31002-31011

40 Compton T, Kurt-Jones EA, Boehme KW, Belko J, Latz E, Golenbock DT, Finberg RW. Human cytomegalovirus activates inflammatory cytokine responses via CD14 and Toll-like receptor 2. J Virol. 2003;77:4588-96

41 Boehme KW, Guerrero M, Compton T. Human cytomegalovirus envelope glycoproteins B and $\mathrm{H}$ are necessary for TLR2 activation in permissive cells. J Immunol. $2006 \mathrm{Nov}$ 15;177(10):7094-102. doi: 10.4049/jimmunol.177.10.7094. PMID: 17082626.

Format:

42 Zdziarski P, Gamian A, Dworacki G. A case report of lymphoid intestitial pneumonia in common variable immunodeficiency: Oligoclonal expansion of effector lymphocytes with preferential cytomegalovirus-specific immune response and lymphoproliferative disease promotion. Medicine (Baltimore). 2017; 96:e7031. doi: 10.1097/MD.0000000000007031. 43Hopkins HA, Monick MM, Hunninghake GW. Cytomegalovirus inhibits CD14 expression on human alveolar macrophages J Infect Dis. 1996;174: 69-74.

${ }^{44}$ Montoya JG, Kogelnik AM, Bhangoo M, Lunn MR, Flamand L, Merrihew LE, Watt T, Kubo JT, Paik J, Desai M. Randomized clinical trial to evaluate the efficacy and safety of valganciclovir in a subset of patients with chronic fatigue syndrome. J Med Virol. 2013 Dec;85(12):2101-9. doi: 10.1002/jmv.23713. Epub 2013 Aug 19. PMID: 23959519. 45 Weiner LM, Li W, Holmes M, et al. Phase I trial of recombinant macrophage colonystimulating factor and recombinant gamma-interferon: toxicity, monocytosis, and clinical effects. Cancer Res. 1994;54: 4084-4090.

46 Daley-Yates PT Inhaled corticosteroids: potency, dose equivalence and therapeutic index Br J Clin Pharmacol. 2015; 80: 372-380.

47 Kim BY, Son Y, Lee J, et al. Dexamethasone inhibits activation of monocytes/macrophages in a milieu rich in 27-oxygenated cholesterol. PLoS One. 2017;12:e0189643. doi:10.1371/journal.pone.0189643

48 Estruch M., Bancells C., Beloki L., Sanchez-Quesada J.L., Ordonez-Llanos J., Benitez S.CD14 and TLR4 mediate cytokine release promoted by electronegative LDL in monocytes." Atherosclerosis 2013; 229:356-362()

49 Jaskula E, Dlubek D, Tarnowska A, Lange J, Mordak-Domagala M, Suchnicki K, Sedzimirska M, Borowik A, Mizia S, Lange A. Anti-CMV-IgG positivity of donors is beneficial for alloHSCT recipients with respect to the better short-term immunological recovery and high level of CD4+CD25high lymphocytes. Viruses. 2015; 7: 1391-408. doi: 10.3390/v7031391.

50 Tamul KR, Schmitz JL, Kane K, Folds JD. Comparison of the effects of Ficoll-Hypaque separation and whole blood lysis on results of immunophenotypic analysis of blood and bone marrow samples from patients with hematologic malignancies. Clin Diagn Lab Immunol $1995 ; 2: 337-342$.

51 Hubl, W., Wolfbauer, G., Andert, S., Thum, G., Streicher, J., Hubner, C., Lapin, A., Bayer, P. M. Toward a new reference method for the leukocyte five-part differential. Cytometry 1997; 30, 72-84.

52 Wirtz PH, von Känel R, Frey K, Ehlert U, Fischer JE. Glucocorticoid sensitivity of circulating monocytes in essential hypertension. Am J Hypertens. 2004;17: 489-494. doi:10.1016/j.amjhyper.2004.01.010 